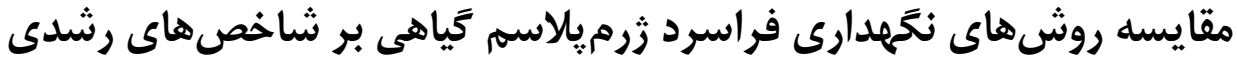 و جوانهزنى بذر كَياه آويشن درني
}

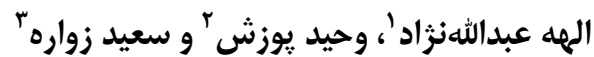

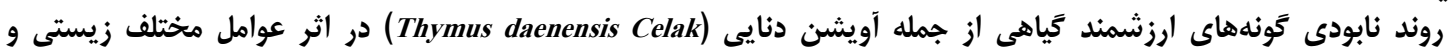

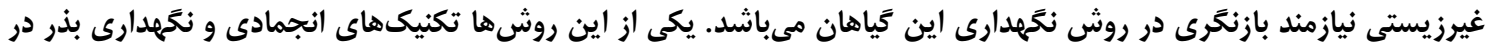

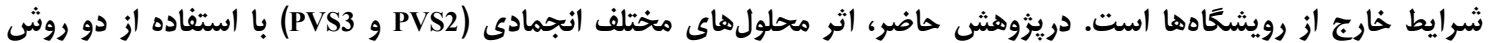

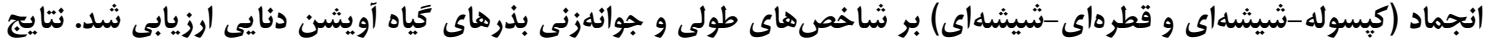

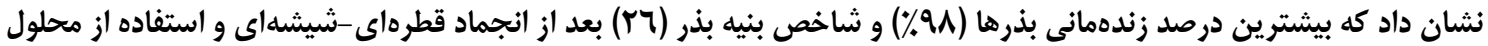

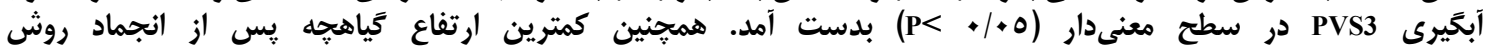

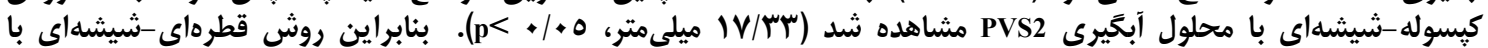

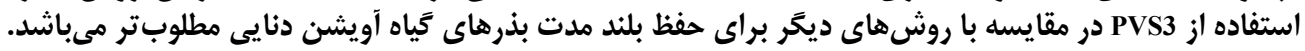

وازههاى كليدى: آويشن دنايى، انجماد شيشهاى، محلول آبخيرى

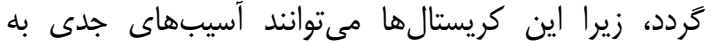

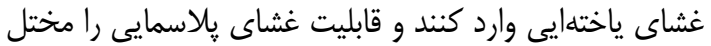

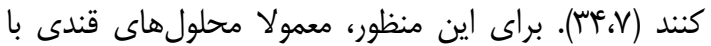

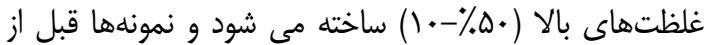

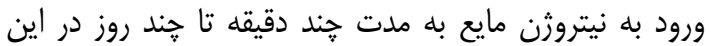

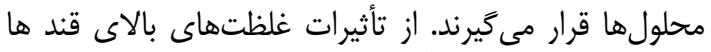

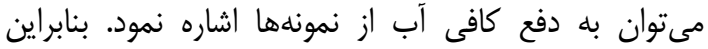

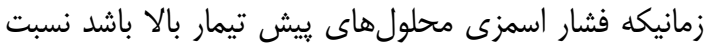

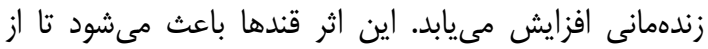

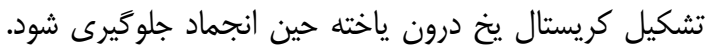

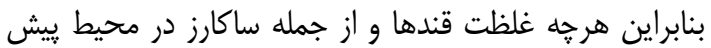

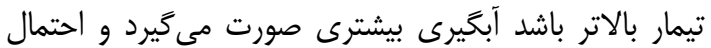

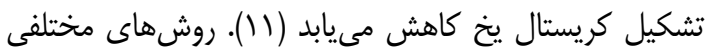

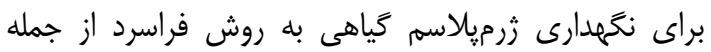

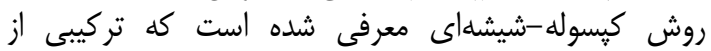

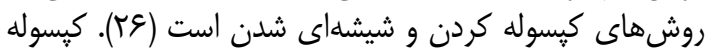

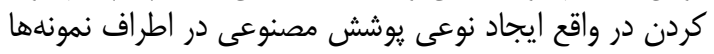

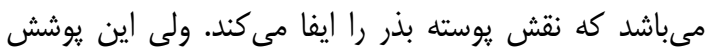

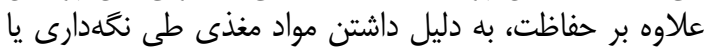

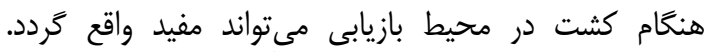

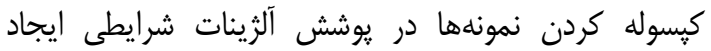

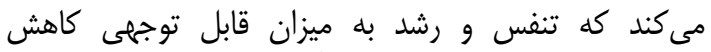

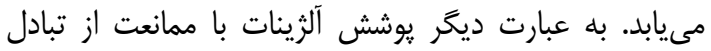

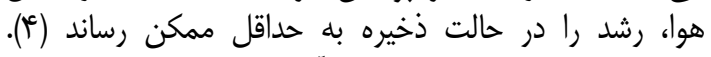

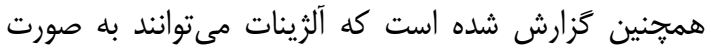

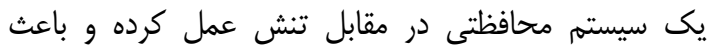

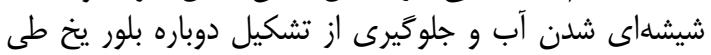

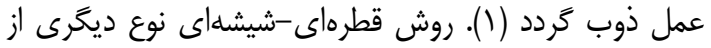

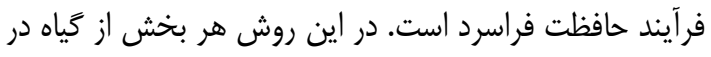

كياهان دارويى و مرتعى به دليل برداشتهاى بـىرويه و

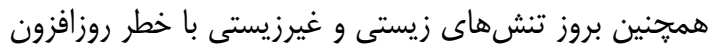

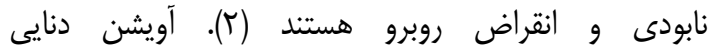
يكى از اين خياهان در النين (Thymus daenensis Celak) معرض نابودى مىباشد (TV). با توجه به اهميت دارويى و

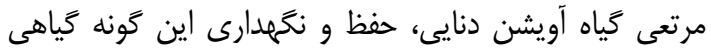

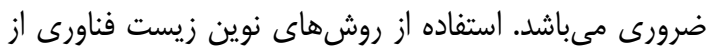

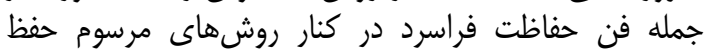

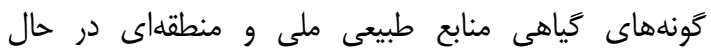

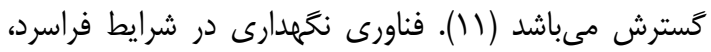
روشى براى ذخيرهسازى بذرها و اندامهاى گياهى

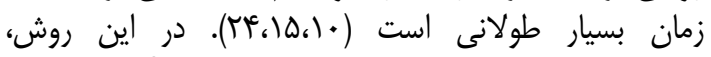

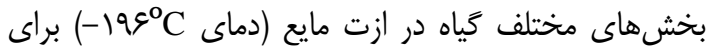

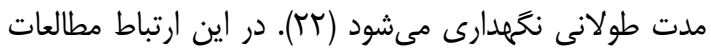

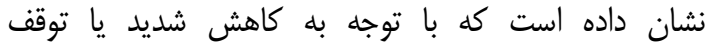

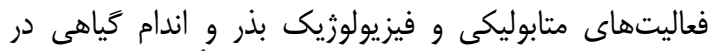

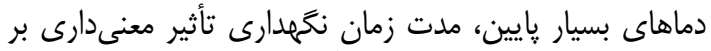

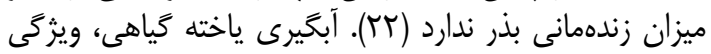

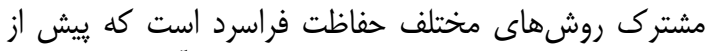

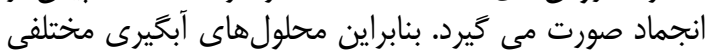

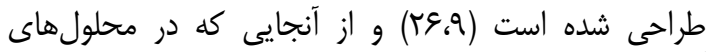

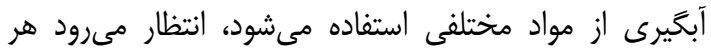

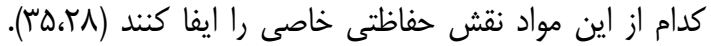

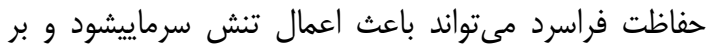

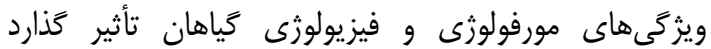

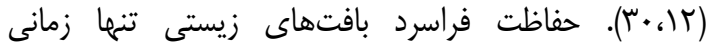

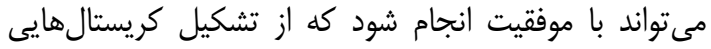

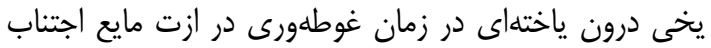




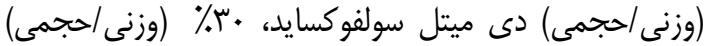

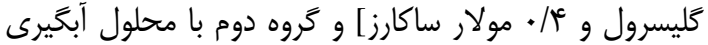

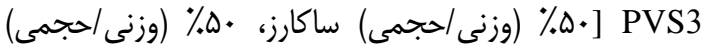

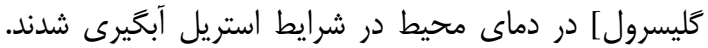

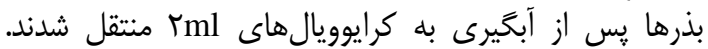

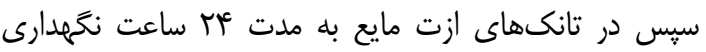

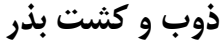

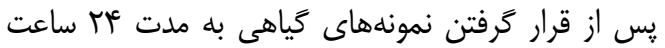

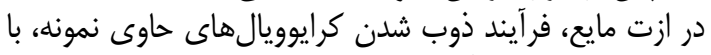

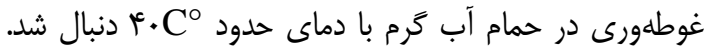

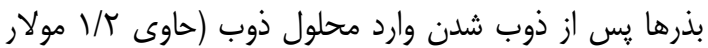

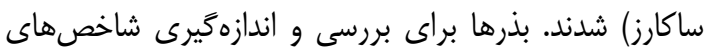

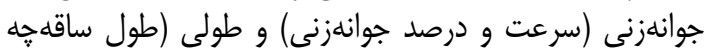

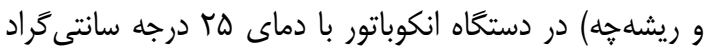

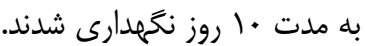

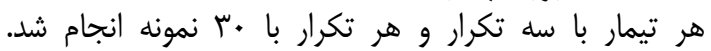

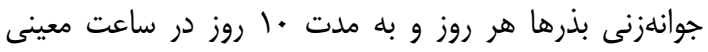

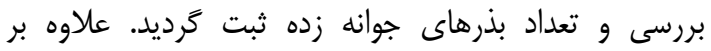

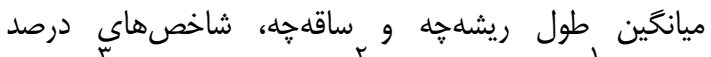

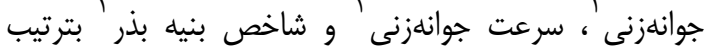

زير محاسبه كَرديدند (بآ). $G P=\frac{\sum N i}{\sum N}$

رابطه (1) هر: مجموع تعداد بذور جوانه زده، Ni: تعداد بذر جوانهانهزده در $G R=\frac{\sum(S i)}{\left.\sum D i\right)}$ هر شمارش، N: تعداد كل بذرها

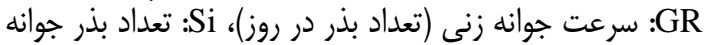

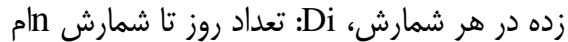

$V I=S L \times G P$

جا Vاخص بنيه بذر، SL: ميانكَين طول كياهجه، GP: درصد

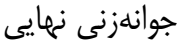
تجزيه و تحليل آمارى نائ آناليز دادهها با نرمافزار SPSS نسخه سب و وروش آناليز

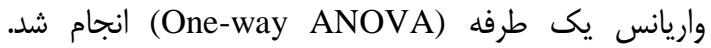
آزمون تكميلى دانكن براى مقايسه ميانكَين دادهانها بين

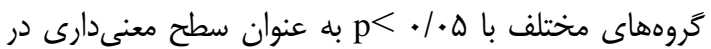

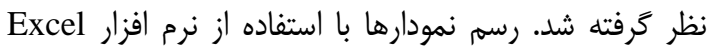

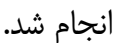

\section{نتايج و بحث}

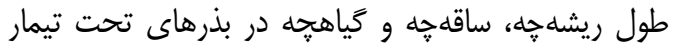

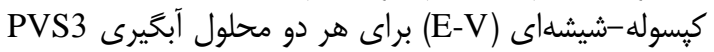

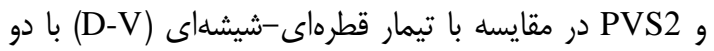

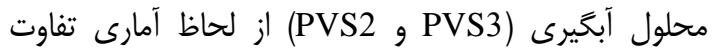

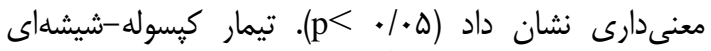

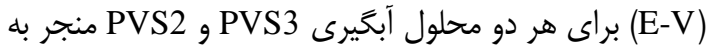

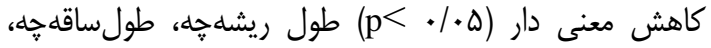
طول كياهجه و شاخص بنيه بذر نسبت به شاهد شد (جدول

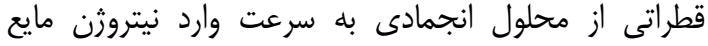

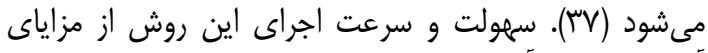

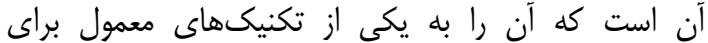

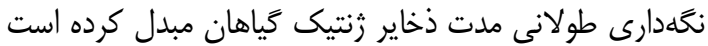

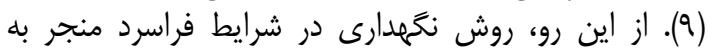

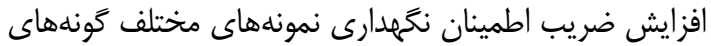

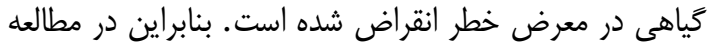

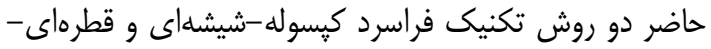

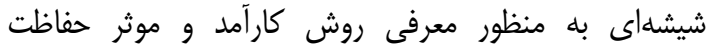
زرريلاسم آويشن دنايى مورد بررسى و مقايسه قرار كرفته

\section{مواد و روشها}

بذرهاى يكنواخت و همكن كَياه آويشن دنايى از شركت

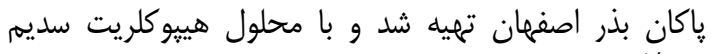

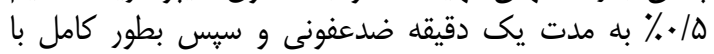

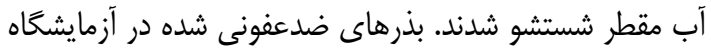

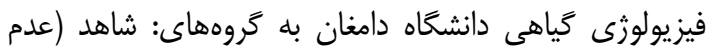

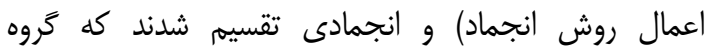

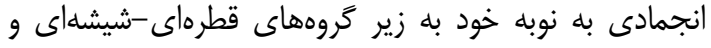
كيسوله-شيشهاى تقسيم شد.

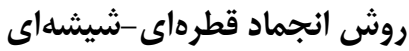

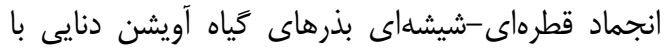

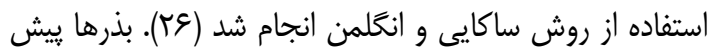

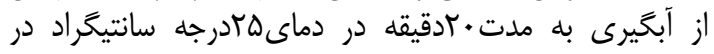

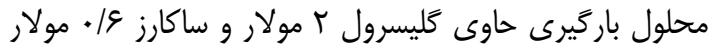

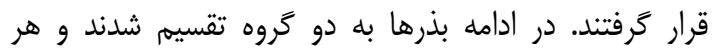

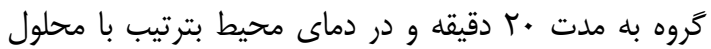

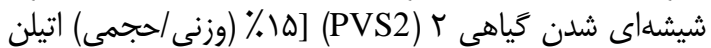

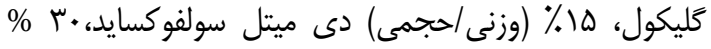

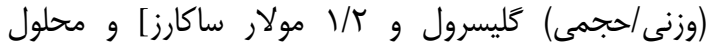

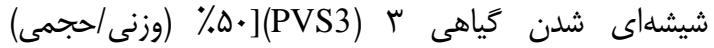

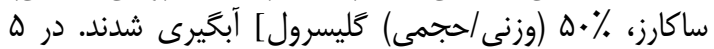

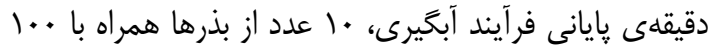

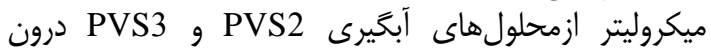

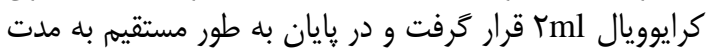

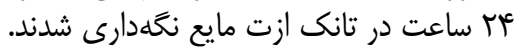
روش انجماد كِّوله

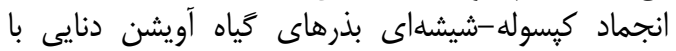

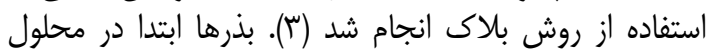

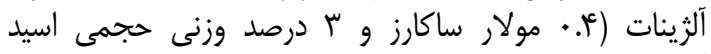

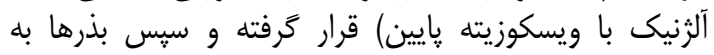

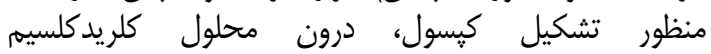

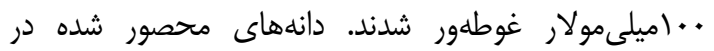

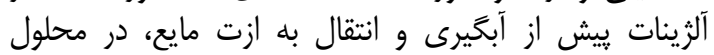

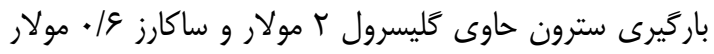

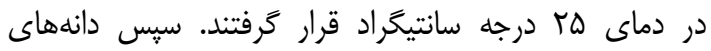

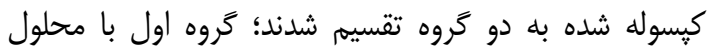

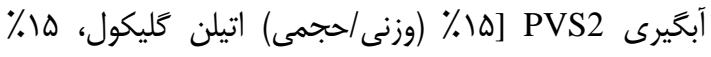


بازيابى اندام هوايى گياه واسابى ڤِ إس از تيمار راس ساقه گياه

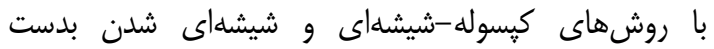

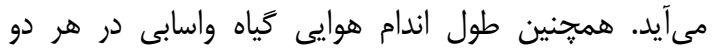

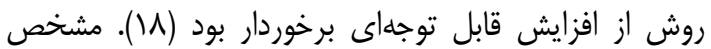

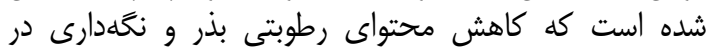

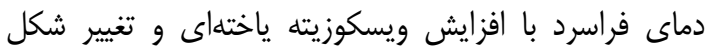

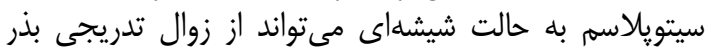

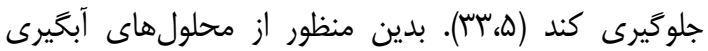

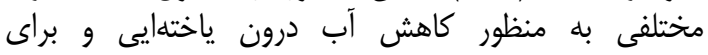

جلوگيرى از تشكيل يخ درون ياخته استفاده مى شود (هاّ).
(). همخنين نسبت طول ريشه קه به ساقه קه در تيمارهاى

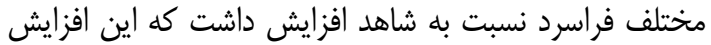

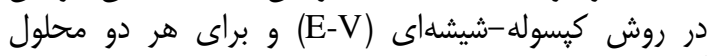
آبخيرى PVS3

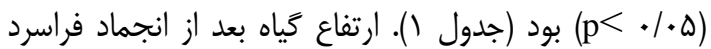

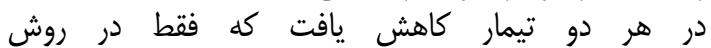
كِيسوله-شيشهاى معنى دار إين = PVS3

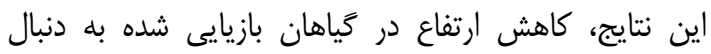

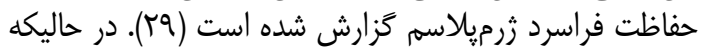

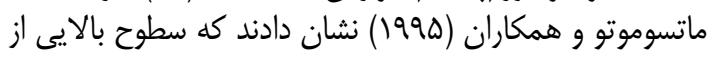

Table 1. The longitudinal indices of Thymus daenensis after recovering of vitrified seeds

جدول ا- شاخصهاى طولى گياه آويشندنايى يّ از بازيابي بذرهاى منجمد شده

\begin{tabular}{|c|c|c|c|c|}
\hline \multicolumn{4}{|c|}{ ميانگين دادهها } & \multirow[b]{2}{*}{ منابع تغييرات } \\
\hline نسبت طول ريشه حه بر ساقه & $\begin{array}{c}\text { طول گياهجه } \\
\text { (mm) }\end{array}$ & طول ريشه & 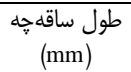 & \\
\hline $0.78 \pm 0.12^{\mathrm{a}}$ & $32.1 \pm 4.11^{\mathrm{a}}$ & $12.99 \pm 2.02^{\mathrm{a}}$ & $16.55 \pm 2.03^{\mathrm{a}}$ & Control \\
\hline $0.88 \pm 0.06^{\mathrm{a}}$ & $26.33 \pm 2.08^{\mathrm{ab}}$ & $12.66 \pm 1.52^{\mathrm{ab}}$ & $14.33 \pm 2.08^{\mathrm{a}}$ & D-Vpvs3 \\
\hline $0.72 \pm 0.17^{\mathrm{a}}$ & $27 \pm 2.64^{\mathrm{ab}}$ & $10.44 \pm 1.26^{\mathrm{ab}}$ & $14.77 \pm 2.45^{\mathrm{a}}$ & D-Vpvs2 \\
\hline $1.53 \pm 0.17^{\mathrm{b}}$ & $21.66 \pm 2.46^{\mathrm{bc}}$ & $12 \pm 1.26^{\mathrm{ab}}$ & $8 \pm 2.45^{\mathrm{b}}$ & E-Vpvs3 \\
\hline $1.55 \pm 0.24^{\mathrm{b}}$ & $17.33 \pm 3.55^{\mathrm{c}}$ & $9.33 \pm 2.08^{\mathrm{b}}$ & $6 \pm 1^{\mathrm{b}}$ & E-Vpvs2 \\
\hline
\end{tabular}

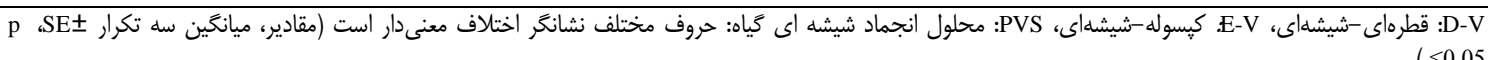

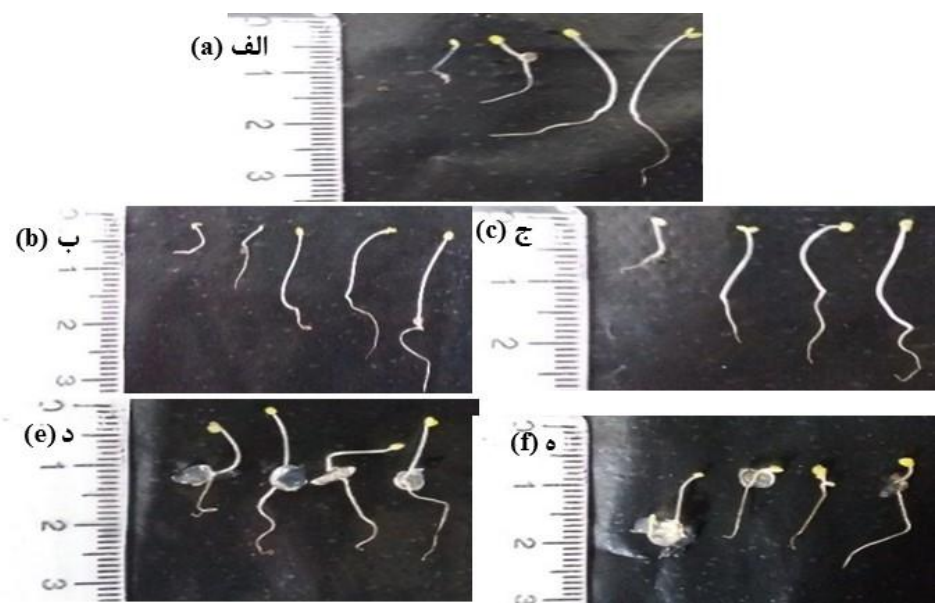

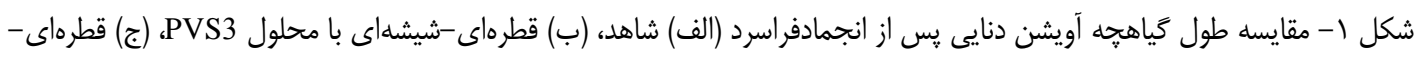

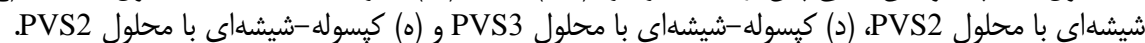

Figure 1 Comparison of Thymus daenensis length after cryopreservation : (a) control, (b) Droplet-vitrification with PVS3, (c) Droplet-vitrification with PVS2, (e) Encapsulation-vitrification with PVS3, (f) Encapsulation-vitrification with PVS2.

كيسوله-شيشهاى با محلول آبخيرى PVS2 بدست آمد. اما

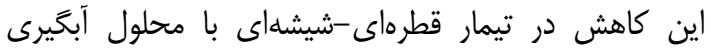
PVS3

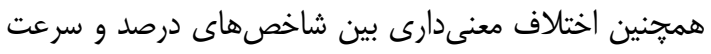

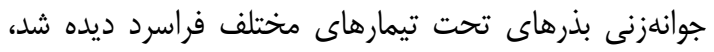

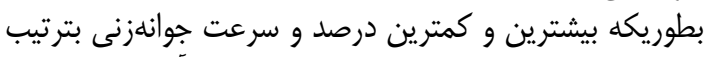

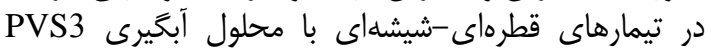

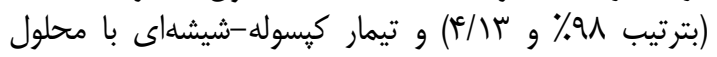

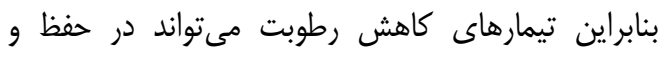

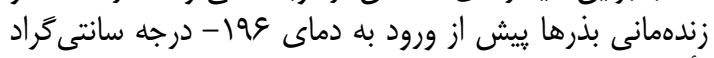

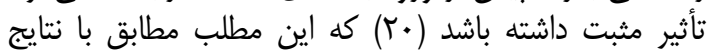
حاصل از تحقيق حاضر مىباشد.

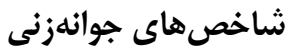
نتايج يزوهش حاضر جوانه نشان داد كه شاخص بنيه بذر تحت

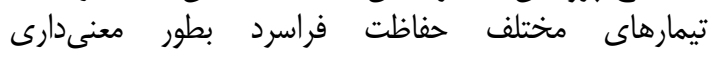
(p) كاهش يافت، بطوريكه بيشترين كاهش در تيمار 
ميزان قوه بذر دارد. درصد بالاى جوانهزنى در روش قطرهاى - مبراي

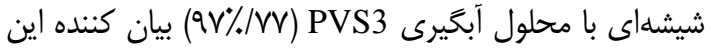

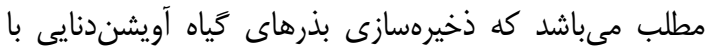

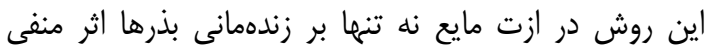

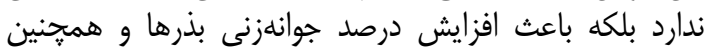

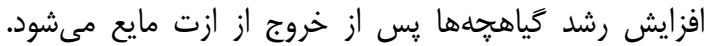

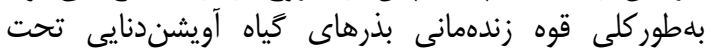

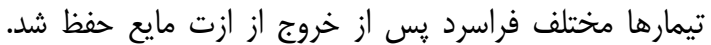

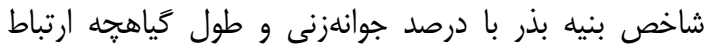

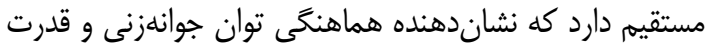

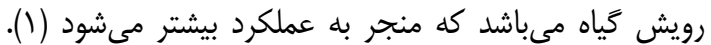
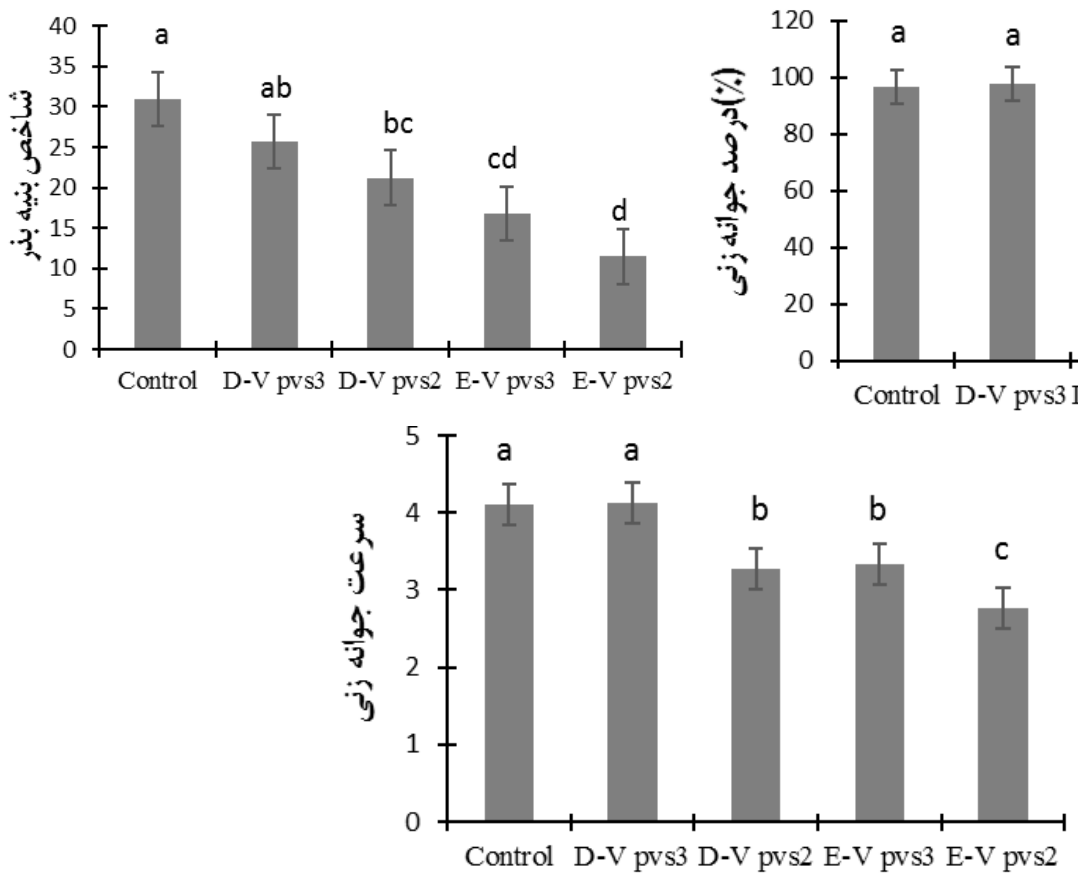

شكل r- بنيه بذر و نرخ جوانهزنى بذرهاى آويشن دنايى بعد از انجماد. حروف مختلف نشانكر تفاوت معنى دار است

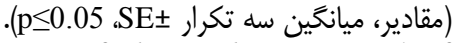

Figure 2. Vigor index and germination rate of Thymus daenensis seeds after cryopreservation. Different letters indicate significant different.(values: mean of three repetitions $\pm S E, p \leq 0.05$ ).
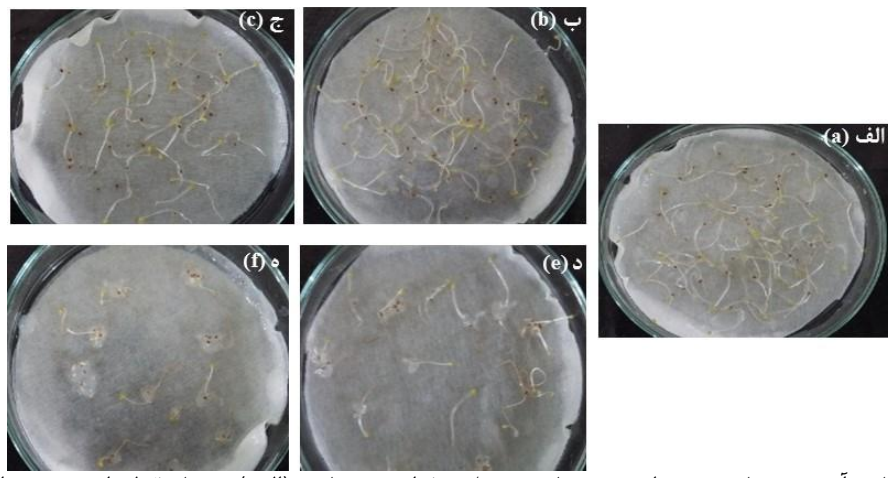

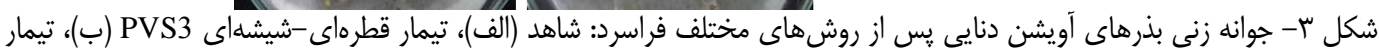

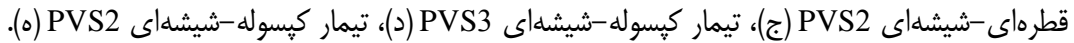

Figure 3. Germination of Thymus daenensis seeds after different cryopreservation methods: (a) control, (b) Dropletvitrification with PVS3, (c) Droplet-vitrification with PVS2, (e) Encapsulation-vitrification with PVS3, (f) Encapsulation-vitrification with PVS2. 


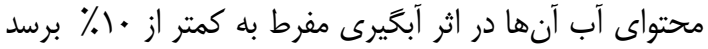

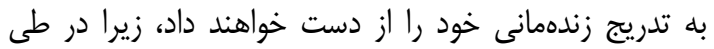

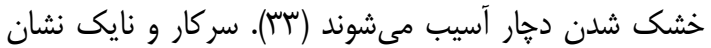

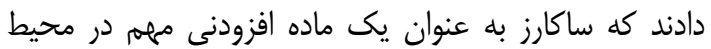

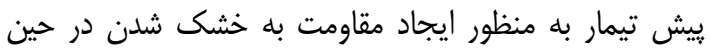

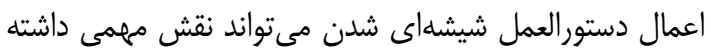

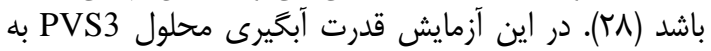

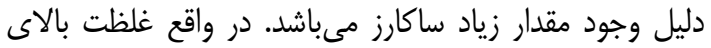

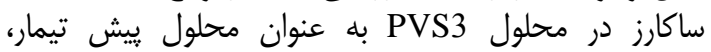

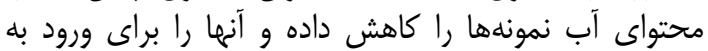

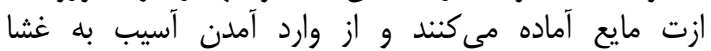

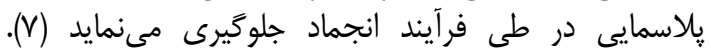

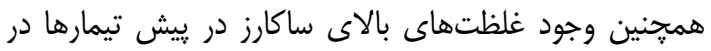

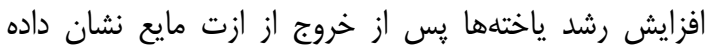

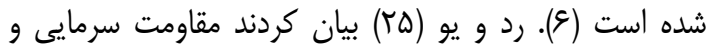

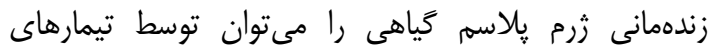

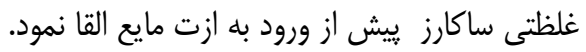

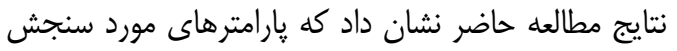

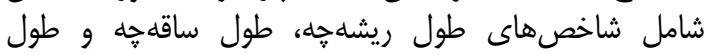

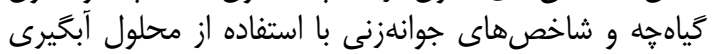

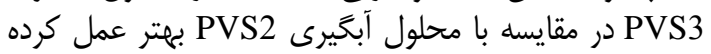

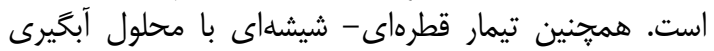

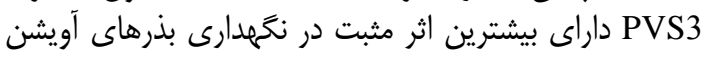

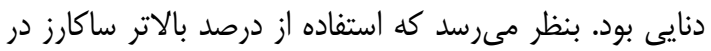

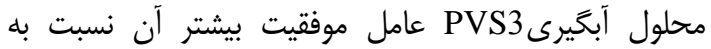

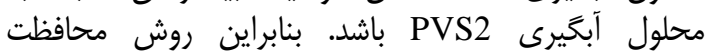
قطرهاى - شيشهاى با محلول آبخيرى PVS

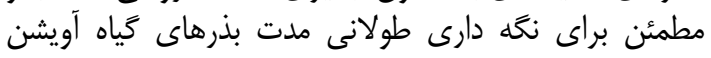
دنايى مىباشد.

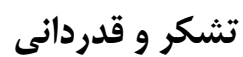

از دانشخاه دامغان بابت حمايت مالى و معنوى دانى يروزه كمال

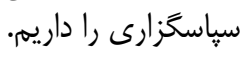

در مطالعهى ديخر مشخص شد كه شاخص قدرت

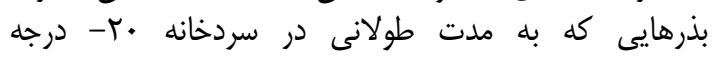

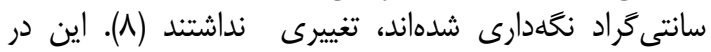

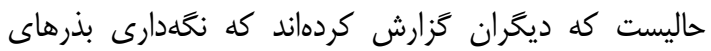

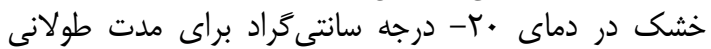

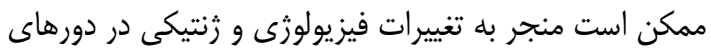

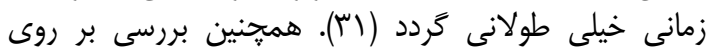

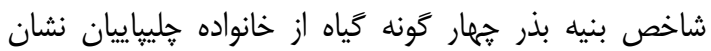

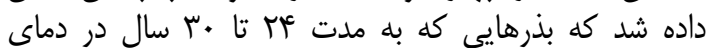

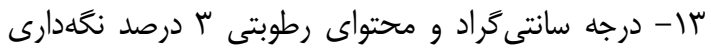

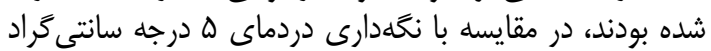

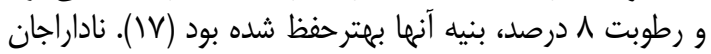

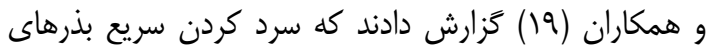

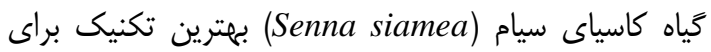
يك انجماد فراسرد است. بطور كلى روش سرد كراس كردن سريع و و

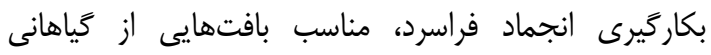

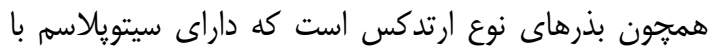

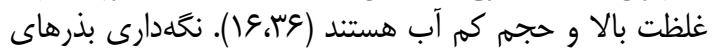

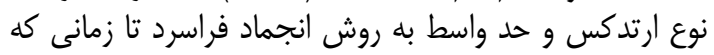

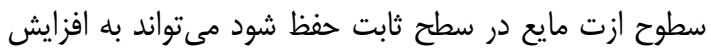

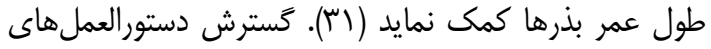

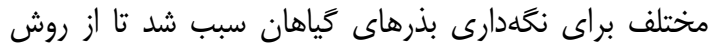

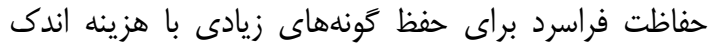

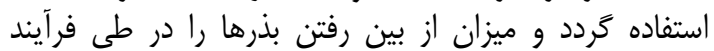

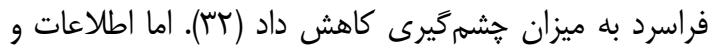

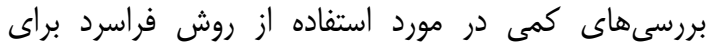

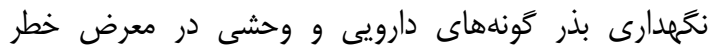

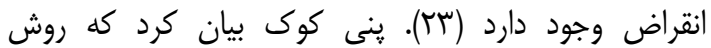

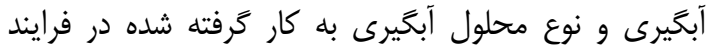

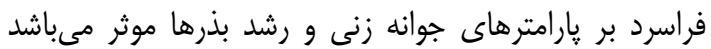

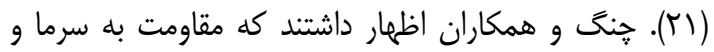

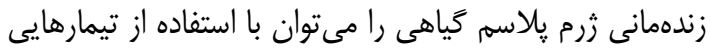

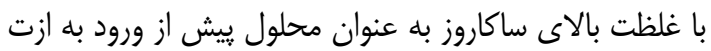

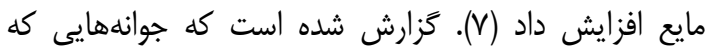

منابع

1. Aryakia, E., H. Ramazani, H. Ghafoori, A. Dolatyari, M.R. Naghavi and S.A. Shahzadeh Fazeli. 2012.The effect of cryopreservation on germination and growth indices of some orthodox seeds. Iranian Journal of Rangelands and Forests Plant Breeding and Genetic Research, 19: 218-230.

2. Arzani, A. 2004. Breeding Field Crops (Authored by: Sleper \& Poehlman). 3th edition,Isfahan University of Technology Publication, $630 \mathrm{pp}$ (In Persian).

3. Block, W. 2003. Water status and thermal analysis of alginate beads used in cryopreservation of plant germplasm. Cryobiology, 47: 59-72.

4. Brodelius, P., L. Linse and K. Nilsson. 1982. Viability and biosynthetic capacity of immobilized plant cells. In Fujiwara A (Ed) plant tissue culture 1982. Proc 5th int. Cong of plant tissue and cell culture Maruzen. Tokyo $371 \mathrm{pp}$.

5. Buitink, J. and O. Leprince. 2008. Intracellular glasses and seed survival in the dry state. Physiologie moléculaire des semences, 331(10): 788-95.

6. Chang, Y. and B.M. Reed. 2000. Extended alternating-temperatur cold acclimation and culture duration improve pear shoot cryopreservation. Cryobiology, 40: 311-322.

7. Chang, Y. and B.M. Reed. 2001. Preculture Conditions Influence Cold Hardiness and Regrowth of Pyrus cordata Shoot Tips after Cryopreservation. HortScience, 36(7): 1329-1333.

8. De Castro Lima, D., A. Sandro Dutra, F. Moura Pontes and F.T. Coelho Bezerra. 2014. Storage of sunflower seeds. Revista Ciência Agronômica, 45(2): 361-369. 
19. مقايسه روشهاى نكمهارى فراسرد زرميلاسم كياهى بر شاخصهاى رشدى و جوانهزنى بذر كياه آويشن دنايى.

9. Engelmann, F. 1997. Importance of desiccation for the cryopreservation of recalcitrant seed and vegetatively propagated species. Plant Genetics Resources Newsletter, 112: 9-18.

10. Engelmann, F. 2004. Plant cryopreservation: Progress and prospects. In Vitro Cellular \& Developmental Biology-Plant, 40: 427-433.

11. Fang, J.Y., A. Wetten and P. Hadiey. 2004. Cryopreservation of cocoa (Theobroma cacao L.) somatic embryos for long-term germplasm storage. Plant Science, 166: 669-675.

12. Ghorbani, A.F. Zarinkamar and A. Fallah. 2009. The Effect of Cold Stress on the Morphologic and Physiologic Characters of Two Rice Varieties in Seedling Stage. Journal of Crop Breeding, 1(3): 5066. (In Persian)

13. Kandil, A.A., A.E. Sharief and E.S.E. Nassar. 2012. Response of some rice (Oryza sativa L.) cultivars to germination under salinity stress. International Journal of Agriculture Sciences, 4: 272-277.

14. Kuleshova, L.L., D.R. Mac Farlane, A.O. Trounson and J.M. Shaw. 1999. Sugars exert a major influence on the vitrification properties of ethylene glycol-based solutions and have low toxicity to embryos and oocytes. Cryobiology, 38(2): 119-130.

15. Lambardi, M., C. Benelli and A. Decarlo. 2005. Cryopreservation as a tool for the long-term conservation of woody plant germplasm: development of the technology at the CNR/INVALSA Institute of Florence. The Role of Biotechnology, 181-182.

16. Li, D.Z. and H.W. Pritchard. 2009. The science and economics of ex situ plant conservation. Trends in Plant Sciences, 14: 614-621.

17. Maselli, F., M.A. Gilabert and C. Conese. 1998. Integration of high and low resolution NDVI data for monitoring vegetation in Mediterranean environments. Remote Sensing of Environment, 63: 208-218.

18. Matsumoto, T., A. Sakai, C. Takahashi and K. Yamada. 1995a. Cryopreservation of in vitro-grown meristems of wasabi (Wasabia japonica) by encapsulation-vitrification method. CryoLetters, 16: 189196.

19. Nadarajan, J., H.J. Staines, E.E. Benson, M. Mansor, B. Krishnapillay and K. Harding. 2006. Optimization of cryopreservation for Sterculia cordata Blume. zygotic embryo using Taguchi experiments. Journal of Tropical Forest Science, 18: 166-172.

20. Ozden-Tokatli, Y., E.A. Ozudogru, F. Gumusel and M. Lambardi. 2007. Cryopreservation of Pistacia spp. seeds by dehydration and one-stepfreezing. CryoLetters, 28(2): 83-94.

21. Pennycooke, J.C. and L.E. Towill. 2000.Cryopreservation of shoot tips from in vitro plantsof sweet potato [Ipomoea batatas (L.) Lam.] by vitrification. Plant Cell Reports, 19: 733-737.

22. Popov, A.S., E.V. Popova, T.V. Nikishina and O.N. Vysotskaya. 2006. Cryobank of plant genetic resources in Russian Academy of Sciences. International Journal of Refrigeration, 29: 403-410.

23. Rajasekharan, P.E. 2006. Prospects of new cryopreservation techniques for conservation oftropical horticultural species. Paper presented at the ICAR Short Course on In Vitro Conservation and Cryopreservation-New Options to Conserve Horticultural Genetic Resources, Banglore, India. 21-30 September.

24. Rao, N.K. 2004. Plant Genetic Resources: Advancing conservation and use through biotechnology. African Journal of Biotechnology, 3: 136-145.

25. Reed, B.M. and X. Yu. 1995. Cryopreservation of in vitro-grown gooseberry and current meristems Cryo-Letters, 16: 131-136.

26. Sakai, A. and F. Engelmann. 2007. Vitrification, encapsulation-vitrification and dropletvitrification: a review. CryoLetters, 28(3): 151-172.

27. Sardari, M., M. Talebi and A. Ghaedi. 2007. Final Report of Phase 2 from Plan of the Collection, Identification, Classification and Herbariums Formation. Research Center of Chaharmahal \& Bakhtiari. (In Persian)

28. Sarkar, D. and P.S. Naik. 1998. Cryopreservation of shoot tips of tetraploid potato (Solanum tuberosum L.) clones by vitrification. Annals of Botany, 82: 455-461.

29. Sershen, B. Varghese, N.W. Pammenter and P. Berjak. 2012. Cryo-tolerance of zygotic embryos from recalcitrant seeds in relation to oxidative stress-A case study on two amaryllid species. Journal of Plant Physiology, 169: 999-1011.

30. Sharifi, P. 2012. Evaluation of cold tolerance of coleoptile growth in some of rice cultivars at germination stage. Journal of Crop Breeding, 4(9): 108-121. (In Persian)

31. Stanwood, P.C. and L.N. Bass. 1981. Seed germplasm preservation using liquid nitrogen. Seed Science and Technology, 9: 423-437.

32. Stanwood, P.C. 1987. Survival of sesame seeds at the temperature $\left(-196^{\circ} \mathrm{C}\right)$ of liquid nitrogen. Crop Science, 27: 327-331.

33. Sun, W.Q., T.C. Irving and A.C. Leopold. 1994. The role of sugar, vitrification and membrane phase transition in seed desiccation tolerance. Physiology Plant, 90: 621-688.

34. Tao, D. and P.H. Li. 1986. Classification of plant cell cryoprotectants. Journal of Theoretical Biology, 123: 305-310.

35. Walters C., G.M. Volk, L.E. Towill and P.H.L. Forsline. 2009. Survival of cryogenically-stored dormant apple buds: a 20 year assessment. Paper presented at the $1^{\text {st }}$ International Symposium on Cryopreservation in Horticultural Species, Leuven, Belgium, 5-9 April 2009.

36. Withers, L.A. 1985. Cryopreservation of cultured plant cells and protoplast. In: Karta, K.K. (ed). Cryopreservation of plant cells and organs. Florida, CRC Press Inc.

37. Wolf, J. and G. Bryant. 1999. Freezing Drying and/or vitrification of membrane-solute-water systems. Cryobiology, 39: 103-129. 


\title{
Comparison of the Different Plant Germplast Cryopreservation Methods on Growth and Germination Indices of Thyme (Thymus Daenensis)
}

\section{Elaheh Abdullahnejad ${ }^{1}$, Vahid Pozesh ${ }^{2}$ and Saeid Zavareh $^{3}$}

\author{
1 and 3- M.Sc. Student and Associated Professor Faculty of Biology and Research Institute of Life Sciences, \\ Damghan University \\ 2- Assistant Professor, Faculty of Biology and Research Institute of Life Sciences, Damghan University, \\ (Corresponding author: poozesh@du.ac.ir)

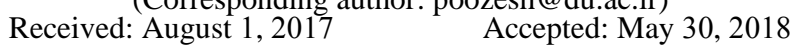

\begin{abstract}
Increasing rate of extinction of valuable plant species such as Thymus daenensis Celak due to various biological and non-biological factors requires an overview of conservation and preservation methods. In this regard, cryopreservation techniques are one of the most important methods for preservation of seeds in non-habitat conditions. In the present study, the effects of different plant vitrification solutions (PVS2 and PVS3) were evaluated using two methods of vitrification (encapsulation-vitrification and droplet-vitrification) on the longitudinal and germination indices of $T$. daenensis seeds. Results showed that the highest viability rate $(98 \%)$ and seed vigor index (26) was obtained after droplet-vitrification method using PVS3 ( $\mathrm{p} \leq 0.05)$. Also, the lowest seedlings height was observed in encapsulation-vitrification method using PVS2 (17/33 mm; $\mathrm{p} \leq 0.05)$. Therefore, droplet-vitrification method using PVS3 is more favorable for the long-term preservation of $T$. daenensis seeds.
\end{abstract}

Keywords: Dehydration Solution, Thymus Daenensis, Vitrification 